\title{
Static domain wall in braneworld gravity
}

\author{
M. C. B. Abdalla ${ }^{1, a}$, P. F. Carlesso ${ }^{1, b}$, J. M. Hoff da Silva ${ }^{2, c}$ \\ ${ }^{1}$ Instituto de Física Teíorica, UNESP, Universidade Estadual Paulista, Rua Dr. Bento Teobaldo Ferraz 271, Bloco II, Barra-Funda, \\ Caixa Postal 70532-2, São Paulo, SP 01156-970, Brazil \\ ${ }^{2}$ Departamento de Física e Química, UNESP, Universidade Estadual Paulista, Av. Dr. Ariberto Pereira da Cunha, 333, Guaratinguetá, SP, Brazil
}

Received: 30 October 2013 / Accepted: 16 December 2013 / Published online: 23 January 2014

(C) The Author(s) 2014. This article is published with open access at Springerlink.com

\begin{abstract}
In this paper we consider a static domain wall inside a 3-brane. Different from the standard achievement obtained in General Relativity, the analysis performed here gives a consistency condition for the existence of static domain walls in a braneworld gravitational scenario. Also the behavior of the domain wall's gravitational field in the newtonian limit is shown.
\end{abstract}

\section{Introduction}

Braneworld models have been thoroughly studied in contemporaneous theoretical physics. This was stimulated by the seminal work of Randall-Sundrum [1,2]. For instance, in Ref. [1] a possible explanation is provided for the hierarchy problem of particle physics. These models are partially inspired by results from string theory that necessarily require the existence of extra dimensions. The braneworld picture may be understood as an effective scenario of the Horava-Witten framework [3], within a warped spacetime. It is interesting to analyze the physical implications of the extra dimensions, whose consequences might include particle physics effects, new astrophysics observables [4], and some cosmological modifications from the standard model. For example, the dark matter problem, raised in the realm of astrophysics and in the cosmology setup, requires particles with no electromagnetic and strong interactions. This last problem can be approached via braneworld scenarios, in which dark matter can be interpreted as massive gravitons from extra dimensions $[5,6]$. Nevertheless, there are many other situations where braneworlds can evoke new insights as regards physical predictions. Some of those situations are the topological defects in cosmology. Those defects may be

\footnotetext{
a e-mail: mabdalla@ift.unesp.br

be-mail: pablofisico@ift.unesp.br

c e-mail: hoff@feg.unesp.br; hoff@ift.unesp.br
}

generated by means of one or several spontaneous symmetry breaking terms in the lagrangian of some scalar field models. In this process, structures as domain walls and cosmic strings may be created at a cosmological scale [7,8]. There are several works studying the gravitational properties of domain walls. Some exhaustive achievements were obtained for the domain wall behavior in the context of the usual theory of general relativity [9-11].

A relevant result, which we are particularly interested in, shows that thick and static domain walls are incompatible with general relativity [12]. It is an appealing and strong result constituting a benchmark in the study of gravitational effects of domain walls. In this work we shall revisit this result in the light of braneworld gravity. In fact, the study of domain walls' gravitational effects within the scope of braneworlds was previously considered [13-17]. Particularly in $[13,14]$, the authors assume a conformal bulk metric, bringing about no significant differences for the domain wall gravitational field from the usual four-dimensional Einstein equation.

Assuming that general relativity holds in the whole fivedimensional bulk, and the four-dimensional brane is endowed with $\mathbb{Z}_{2}$ symmetry, one can recover the gravitational equation on the brane in a suitable and precise way. The result stands for corrections coming from the extra dimension (encoded in the Weyl tensor), as well as some modifications proportional to the square of the stress tensor $[18,19]$. For the specific case investigated in this paper, the contributions from the square of the stress tensor are identically zero, and all the modifications rest upon the Weyl tensor term. As we shall see, the Weyl term, under very general assumptions, is responsible for allowing the existence of static domain walls in the braneworld. This result is in acute contrast with the one obtained within the usual general relativity.

This paper is organized as follows: in Section 2, we briefly review the main steps leading to an effective (and modified) gravitational field equation on the brane. Going further, we show that static domain walls are allowed in the braneworld 
gravity. In the final section we conclude, giving a simple physical interpretation and pointing out some perspectives in this branch of research.

\section{Braneworld gravity}

As previously mentioned, the extra-dimension model considered here is to a quite large extent based on the RandallSundrum models (mostly [2]). Therefore, we consider a 3brane, in which the standard model particles are localized, embedded in a five-dimensional bulk with a $\mathbb{Z}_{2}$ symmetric fifth dimension. That scenario provides a distinct description for the gravitational dynamics. To achieve the gravitational equations on the brane, it is taken for granted that Einstein's field equations hold in five dimensions. Then, through the Gauss-Codazzi formalism and Israel matching conditions it is possible to obtain the gravitational equations $[18,20]$ relating the intrinsic brane quantities (metric $g_{\mu \nu}$ and Einstein's $G_{\mu \nu}$ tensors) with the brane stress-energy tensor $\tau_{\mu \nu}$ :

$G_{\mu \nu}=-\Lambda g_{\mu \nu}+\kappa_{4}^{2} \tau_{\mu \nu}+\kappa_{5}^{4} \Pi_{\mu \nu}-E_{\mu \nu}$,

where $\Lambda=\frac{\kappa_{5}^{2}}{2}\left(\Lambda_{5}+\kappa_{5}^{2} \lambda_{b}^{2} / 6\right)$ is an effective brane cosmological constant (determined by the bulk cosmological constant $\Lambda_{5}$ and the intrinsic brane tension $\lambda_{b}$ ). These equations are closely related with the Einstein field equations; the distinction lies with the two last terms on the right-hand side. The tensor $\Pi_{\mu \nu}$ is given by

$\Pi_{\mu \nu}=-\frac{1}{4} \tau_{\mu}^{\sigma} \tau_{\nu \sigma}+\frac{1}{12} \tau \tau_{\mu \nu}+\frac{1}{8} q_{\mu \nu} \tau_{\alpha \beta} \tau^{\alpha \beta}-\frac{1}{24} q_{\mu \nu} \tau^{2}$,

which is fully dependent on the brane stress-energy tensor. Otherwise, the term $E_{\mu \nu}$ (the so-called Weyl fluid) is a nonlocal term, depending on the bulk's Weyl tensor. As we shall see, it is this tensor that is responsible for and enables the existence of static domain walls in braneworld gravity.

\section{Static domain walls}

Let us start by outlining the basic formalism describing domain walls. These structures can be generated by a scalar field lagrangian like

$L=\frac{1}{2} \partial_{\mu} \phi \partial^{\mu} \phi-\lambda^{2}\left(\phi^{2}-\eta^{2}\right)^{2}$.

It is the origin of a topological anomaly on the transition layer between the two vacuum states associated with the potential of the above lagrangian. If we consider a dependence $\phi=\phi(x)$ (where $x$ is parameterizing one of the spatial dimensions) the classical field equations give $\phi(x)=\eta \tanh (\sqrt{2} \lambda \eta x)$.

By assuming the spacetime nearly Minkowskian, we determine the stress-energy tensor for the scalar field lagrangian (3):

$T_{\mu \nu}=\partial_{\mu} \phi \partial_{\nu} \phi-\eta_{\mu \nu}\left[\frac{1}{2} \partial_{\alpha} \phi \partial^{\alpha} \phi-\lambda^{2}\left(\phi^{2}-\eta^{2}\right)^{2}\right]$.

For the scalar field given by (4), we compute the stress-energy components, so that we rewrite the stress-energy tensor as

$T_{v}^{\mu}=\sigma(x)\left(\delta_{v}^{\mu}+\xi^{\mu} \xi_{v}\right)$

where $\xi^{\mu}$ is a unit spacelike vector orthogonal to the wall surface and

$\left.\sigma(x)=2 \lambda^{2} \eta^{4}[\cosh (\sqrt{2} \lambda \eta x))\right]^{-4}$.

The above relations show how the energy for the scalar field is distributed in the spacetime. The function $\sigma(x)$ has a peak centered at $x=0$, characterizing the domain wall, whose thickness is determined by the relation $\delta \sim \frac{1}{\lambda \eta}$.

Many works dealing with domain walls in general relativity consider the thin-case limit, utilizing the Dirac delta function to localize the domain wall $[9,10]$. In this work, we consider a thick domain wall where the energy distribution in spacetime is accounted for by (6) along with (7).

As seen in the expression (6), the contraction of $\xi^{\mu}$ with $T_{v}^{\mu}$ is null. Therefore, contracting Eq. (5) with $\xi^{\mu}$ gives

$\xi^{\mu} \partial_{\mu} \phi \partial_{\nu} \phi-\xi_{\nu}\left[\frac{1}{2} \partial_{\mu} \phi \partial^{\mu} \phi-\lambda^{2}\left(\phi^{2}-\eta^{2}\right)^{2}\right]=0$.

The above expression shows that $\partial_{\nu} \phi$ is proportional to $\xi_{\nu}$ and therefore its a hypersurface orthogonal vector implying the relation $\nabla_{\mu} \xi_{\nu}-\nabla_{\nu} \xi_{\mu}=0$. By comparing the expressions for $T_{v}^{\mu}$, (5), and (6), we obtain the relation

$\sigma=2 \lambda^{2}\left(\phi^{2}-\eta^{2}\right)^{2}$.

Taking the partial derivative in the above equation, we conclude that

$\partial_{\alpha} \sigma=\mathcal{N} \xi_{\alpha}$

where $\mathcal{N}$ is a scalar. The energy conservation condition over $T_{v}^{\mu}$ in Eq. (6) gives

$\nabla_{\nu} \sigma+\nabla_{\mu} \xi^{\mu} \xi_{v}+\sigma \nabla_{\mu} \xi^{\mu}+\sigma \xi^{\mu} \nabla_{\mu} \xi_{v}=0$.

By contracting the above equation with $\xi^{\nu}$ and taking into account that here we have a unit vector (which implies $\left.\xi^{\nu} \nabla_{\mu} \xi_{\nu}=0\right)$, we have 
$\nabla_{\mu} \xi^{\mu}=0$

Writing the Riemann tensor as

$\nabla_{\alpha} \nabla_{\beta} \xi^{\mu}-\nabla_{\beta} \nabla_{\alpha} \xi^{\mu}=R_{\lambda \alpha \beta}^{\mu} \xi^{\lambda}$

we use the expression (12) in the above equation to obtain a relation for the Ricci tensor:

$S_{\alpha \beta} S^{\alpha \beta}+R_{\alpha \beta} \xi^{\alpha} \xi^{\beta}=0$

where

$S_{\alpha \beta} \equiv \nabla_{\alpha} \xi_{\beta}$

Braneworld gravitation, given by means of the expression (1), allows us to determine an expression for the Ricci tensor. Meanwhile, first we compute the $\Pi_{\mu \nu}$ (2) term, by means of the stress-energy tensor for the domain wall spacetime. It can be readily verified that the result is $\Pi_{\mu v}=0$ and therefore we only have

$R_{\mu \nu}-\frac{1}{2} g_{\mu \nu} R=8 \pi T_{\mu \nu}-\Lambda g_{\mu \nu}-E_{\mu \nu}$.

Combining this relation with the expression (14), we find the consistency condition for the static domain walls in braneworld gravitation,

$S_{\alpha \beta} S^{\alpha \beta}+12 \pi \sigma=\Lambda+E_{\mu \nu} \xi^{\mu} \xi^{\nu}$.

Now, we perform an analysis of this consistency condition, in two parts.

A. The case of general relativity

As a first step, we consider the absence of the cosmological constant term as well as the contribution from the extra dimension $E_{\mu \nu} \xi^{\mu} \xi^{\nu}$. In this case, Eq. (17) becomes

$S_{\alpha \beta} S^{\alpha \beta}+12 \pi \sigma=0$,

that is, we recover the consistency condition from standard general relativity without cosmological constant [12]. Once $\sigma>0$, it is necessary that $S_{\alpha \beta} S^{\alpha \beta}<0$ to satisfy the condition for the existence of static domain walls in general relativity.

However, for the product $S_{\alpha \beta} S^{\alpha \beta}$ to be negative valued, it is necessary that $S_{\alpha \beta}$ have complex eigenvalues [21]. This fact implies a pair of complex conjugate eigenvectors. However, we know that $S_{\alpha \beta}$ has at least two real eigenvalues (because $S_{\alpha}^{\alpha}=0$ and $S_{\alpha \beta} \xi^{\alpha}=0$ ).

The condition of staticity for the domain wall implies the existence of a timelike hypersurface orthogonal Killing vector. The Lie derivative over the stress-energy tensor (6) along the Killing vector field vanishes, implying

$\mathcal{L}_{K}(3 \sigma)=0 \rightarrow K^{\alpha} \partial_{\alpha} \sigma=0$ and

$K^{\mu} \nabla_{\mu} \xi_{\beta}=\xi^{\mu} \nabla_{\mu} K_{\beta}$

Taking into account Eq. (10) in (19), we have

$\xi^{\alpha} K_{\alpha}=0$.

Hypersurface orthogonality implies

$K_{[\alpha} \nabla_{\gamma} K_{\beta]}=0$,

or

$\xi^{\alpha} K_{[\alpha} \nabla_{\gamma} K_{\beta]}=0$.

By means of Eqs. (20) and (21), we obtain

$K_{\beta} K^{\alpha} \nabla_{\alpha} \xi_{\gamma}-K_{\gamma} K^{\alpha} \nabla_{\alpha} \xi_{\beta}=0$

or, contracting with $K^{\beta}$,

$K^{\alpha} S_{\alpha \gamma}=\rho K_{\gamma}$,

where $\rho=\left(K^{\alpha} K^{\beta} \nabla_{\alpha} K_{\beta}\right) / K^{\beta} K_{\beta}$.

Equation (25) shows that $K^{\alpha}$ is an eigenvector of $S_{\alpha \gamma}$. This fact precludes the existence of a pair of complex conjugate eigenvectors for $S_{\alpha \gamma}$; thus the consistency condition (18) is not satisfied and models of static domain walls are incompatible with general relativity [12].

It is noteworthy that the authors in [12] have not considered the influence of the cosmological constant on Einstein's equations to obtain this result (perhaps because the cosmological constant was just a theoretical hypothesis when that article was written). If now we consider the influence of the cosmological constant, a new condition arises:

$S_{\alpha \beta} S^{\alpha \beta}+12 \pi \sigma=\Lambda$

As both terms on the left-hand side of the above equation are positive, it is necessary that both have the same order of the cosmological constant, or $\lambda^{2} \eta^{4} \sim \Lambda$, leading to a very large thickness for the domain wall.

B. The braneworld case

The relation (17) obtained above constrains the Weyl fluid term. In order to clarify the influence of the Weyl fluid on the domain wall, we write it here in a cosmological fluid form:

$E_{\mu \nu}=-k^{4}\left[U\left(u_{\mu} u_{\nu}-\frac{1}{3} h_{\mu \nu}\right)+P_{\mu \nu}+Q_{(\mu} u_{\nu)}\right]$,

where we decompose the metric tensor by means of a 4velocity field $\left(g_{\mu \nu}=h_{\mu \nu}+u_{\mu} u_{v}\right) . U=-k^{-4} E_{\mu \nu} u^{\mu} u^{v}$ represents the dark radiation component, $P_{\mu \nu}=-k^{-4}\left[h_{(\mu}^{\alpha} h_{v)}^{\beta}-\right.$ 
$\left.\frac{1}{3} h_{\mu \nu} h^{\alpha \beta}\right] E_{\alpha \beta}$ is the anisotropic pressure and $Q_{\mu}=-k^{-4} h_{\mu}^{\alpha}$ $E_{\alpha \beta} u^{\beta}$ the energy flux associated with the Weyl dark fluid.

The staticity condition for the domain wall spacetime requires a null energy flux. Considering the case of a planar domain wall, to simplify the analysis, $S_{\mu \nu}$ vanishes and the constraint (17) becomes

$12 \pi \sigma=E_{\mu \nu} \xi^{\mu} \xi^{\nu}$

Unlike the consistency condition resulting from general relativity, (14), Eqs. (17) and (28) from the braneworld scenario are not necessarily in conflict with the presence of static domain walls. Those relations restrain just the Weyl fluid components along the transversal direction to the domain wall. In terms of dark fluid components, Eq. (28) gives

$P_{\mu \nu} \xi^{\mu} \xi^{\nu}=\frac{1}{3} U(x)-12 \pi k^{-4} \sigma(x)$.

Plugging this relation into (30), we obtain

$$
\begin{aligned}
E_{\mu \nu}=-k^{4}[ & U\left(u_{\mu} u_{\nu}-\frac{1}{3} w_{\mu \nu}\right) \\
& \left.+12 \pi k^{-4} \sigma(x) \xi_{\mu} \xi_{\nu}+w_{\mu}^{\alpha} P_{\alpha \nu}\right],
\end{aligned}
$$

where $w_{\mu \nu}$ is orthogonal to $u^{\mu}$ and $\xi^{\mu}$, these being related by $\left(g_{\mu \nu}=w_{\mu \nu}+u_{\mu} u_{v}-\xi_{\mu} \xi_{v}\right)$.

Let us analyze the immediate consequences of this model. For a stress-energy tensor of the form $T_{\mu}^{\nu}=\operatorname{diag}\left(\rho-P_{1}-\right.$ $\left.P_{2}-P_{3}\right)$, the newtonian limit over Einstein's equations give

$\nabla^{2} \phi=4 \pi\left(\rho+P_{1}+P_{2}+P_{3}\right)$.

The usual attractive matter distribution in spacetime, in the newtonian limit, is described by Poisson's equation, $\nabla^{2} \phi=$ $4 \pi \rho$, where the pressure terms are neglected. In that model, taking the diagonal contribution of dark fluid components together with the domain wall stress-energy tensor, we obtain

$\nabla^{2} \phi=4 \pi\left(2 k^{4} U-\sigma\right)$

In the absence of dark fluid components, we recover the result of Einstein's gravitation in the newtonian limit [9], in which the domain wall exhibits a repulsive gravitational force over test particles. Nevertheless, in the braneworld scenario, the Weyl fluid brings other possibilities for the domain wall gravitation. Depending on the value for the dark fluid components, the wall can produce either an attractive or a repulsive gravitational field, or, in a fine tuning, even a null gravitational influence.

\section{Final remarks}

The main result of this paper is that we have shown that the braneworld picture can have static domain walls, although general relativity prohibits these structures. Also, we see that static domain walls within the braneworld context can have different properties, like an attractive gravitational field. In fact, it is well known that domain walls can be used to distinguish between modified gravity theories and general relativity [22].

An interesting result of the domain wall gravitation in braneworld is that the stress-energy tensor of the wall does not have any extra influence over the gravitational dynamics if compared with Einstein's gravity. It occurs because the term $\Pi_{\mu \nu}$ on the right-hand side of (1) vanishes for the domain wall stress-energy tensor. The unique extra contribution performed by the braneworld model is due to the Weyl fluid term. Meanwhile, if we consider a conformal metric for the bulk spacetime, the Weyl tensor vanishes, as does the Weyl fluid term in Eq. (1). In that case, we recover the result of $[13,14]$, where there is no difference between Einstein's gravity and braneworld gravity for the domain wall spacetime. In the case of general relativity, to probe the inconsistency of static domain walls [12] an energy distribution is considered over the nearly Minkowski spacetime, and staticity of the spacetime is assumed for the domain wall. In this way, it is reasonable to expect that the system no longer holds on to Minkowski spacetime, since in fact we do have a gravitational source. In the braneworld context, however, the dark fluid offsets the influence of the stress-energy of the domain wall in such a manner that it becomes possible that a static domain wall appears in this context.

Let us finalize by observing that in this work we have implemented the so-called brane-based formalism, in which the 3-brane is localized on a point of extra dimension and, by means of Gauss-Codazzi equations and junction conditions, it is possible to obtain the brane's effective gravity. Such an approach leaves undetermined the bulk contribution over braneworld gravitation (encoded by means of the dark fluid term in Eq. (30)). That indeterminacy over the Weyl fluid form allows us to constrain some components, so that the staticity condition is satisfied for the existence of static domain walls in braneworld scenarios. Therefore, this method restricts the bulk gravitation. There is another approach in which we firstly determine the bulk metric properties, the so-called bulk-based formalism. For a spherically vacuum bulk spacetime, a generalization of the Birkhoff theorem [23] states that the metric bulk represents a Schwarzschild-AdS spacetime. For this case, a moving brane in the bulk represents an expanding universe. Taking a bulk Schwarzschild-AdS metric, it was demonstrated [5] that the Weyl fluid components behave as follows: the dark pressure term is null, while the dark radiation (associated 
with the bulk black hole mass) is fairly constant. Therefore, in view of Eqs. (28) and (29), we conclude that static domain walls are incompatible with the metrics of SchwarzschildAdS type for the bulk, since the staticity condition cannot be fulfilled in this case.

Acknowledgments M. C. B. Abdalla and J. M. Hoff da Silva acknowledge to Conselho Nacional de Desenvolvimento Científico e Tecnológico (CNPq) for partial financial support (482043/2011-3; 308623/2012-6). P. F. Carlesso acknowledges to CAPES-Brazil for financial support.

Open Access This article is distributed under the terms of the Creative Commons Attribution License which permits any use, distribution, and reproduction in any medium, provided the original author(s) and the source are credited.

Article funded by $\mathrm{SCOAP}^{3}$ and licensed under CC BY 4.0

\section{References}

1. L. Randall, R. Sundrum, Phys. Rev. Lett. 83, 3370 (1999)

2. L. Randall, R. Sundrum, Phys. Rev. Lett. 83, 4690 (1999)

3. P. Horava, E. Witten, Nucl. Phys. B 460, 506 (1996)

4. R. Maartens, Living Rev. Relativity 7, 7 (2004)
5. R. Maartens, in Reference Frames and Gravitomagnetism, ed. by J. Pascual-Sanchez et al. (World Sci., 2001), p. 93

6. M.K. Mak, T. Harko, Phys. Rev. D 70, 024010 (2004)

7. Y.B. Zel'dovich, I.Y. Kobzarev, L.B. Okun', Sov. Phys. JETP 40, 1 (1974)

8. T.W.B. Kibble, J. Phys. A Math. Gen. 9, 1387 (1976)

9. A. Vilenkin, Phys. Lett. 133B, 177 (1983)

10. J. Ipser, P. Sikivie, Phys. Rev. D 30, 084013 (2002)

11. L.M. Widrow, Phys. Rev. D 39, 3571 (1989)

12. A.K. Raychaudhuri, G. Mukherjee, Phys. Rev. Lett. 59, 1504 (1987)

13. R. Gregory, A. Padilla, Phys. Rev. D 65, 084013 (2002)

14. R. Gregory, A. Padilla, Class. Quant. Grav. 19, 279 (2002)

15. R. Gregory, Phys. Rev. D 43, 520 (1991)

16. V. Silveira, M.D. Maia, Phys. Lett. A 174, 280 (1993)

17. A.L. Larsen, Phys. Lett. A 181, 369 (1993)

18. T. Shiromizu, K. Maeda, M. Sasaki, Phys. Rev. D 62, 043523 (2000)

19. A.N. Aliev, A.E. Gumrukcuoglu, Class. Quant. Grav. 21, 5081 (2004)

20. F. Bonjour, C. Charmousis, R. Gregory, Phys. Rev. D 62, 083504 (2000)

21. L.P. Eisenhart, Riemannian Geometry (Princeton University Press, Princeton, 1964)

22. G. Dvali, G. Gabadadze, O. Pujolas, R. Rahman, Phys. Rev. D 75, 124013 (2007)

23. P. Bowcock, C. Charmousis, R. Gregory, Class. Quant. Grav. 17, $4745(2000)$ 